Publications of the Astronomical Society of the Pacific

97:616-633, July 1985

\title{
THE BRIGHTEST HIGH-LATITUDE 12-MICRON IRAS * SOURCES
}

\author{
P. HACKING
}

Jet Propulsion Laboratory, California Institute of Technology, 4800 Oak Grove Drive, Pasadena, California 91103

\section{G. NEUGEBAUER}

California Institute of Technology, Downes Lab 320-47, Pasadena, California 91125

\section{J. EMERSON}

Queen Mary College, London, United Kingdom

C. BEICHMAN AND T. CHESTER

Jet Propulsion Laboratory, California Institute of Technology, 4800 Oak Grove Drive, Pasadena, California 91103

$$
\text { F. GILLETT }
$$

Kitt Peak National Observatory, National Optical Astronomy Observatories, $\dagger$ P.O. Box 26732, Tucson, Arizona 85726-6732

\section{H. HABING}

Sterrewacht Leiden, P.O. Box 9513, 2300 RA, Leiden, The Netherlands

G. HELOU

Jet Propulsion Laboratory, California Institute of Technology, 4800 Oak Grove Drive, Pasadena, California 91103

J. HOUCK

Cornell University, Ithaca, New York 14853

F. OLNON

Stichting voor Ruimteonderzeok, Leiden, The Netherlands

M. ROWAN-ROBINSON

Queen Mary College, London, United Kingdom

B. T. SOIFER

California Institute of Technology, Downes Lab 320-47, Pasadena, California 91125

\author{
AND \\ D. WALKER \\ Queen Mary College, London, United Kingdom \\ Received 1985 January 2 , revised 1985 April 11
}

\begin{abstract}
The Infrared Astronomical Satellite (IRAS) Point Source catalog was searched for sources brighter than $28 \mathrm{Jy}(0 \mathrm{mag})$ at $12 \mu \mathrm{m}$ with absolute galactic latitude greater than $30^{\circ}$ excluding the Large Magellanic Cloud. The search resulted in 269 sources, two of which are the galaxies NGC 1068 and M82. The remaining 267 sources are identified with, or have infrared color indices consistent with late-type stars some of which show evidence of circumstellar dust shells. Seven sources are previously uncataloged stars. K and M stars without circumstellar dust shells, M stars with circumstellar dust shells, and carbon stars occupy well-defined regions of infrared color-color diagrams.
\end{abstract}

Key words: infrared sources-late-type stars-circumstellar matter

*The Infrared Astronomical Satellite was developed and is operated by the U.S. National Aeronautics and Space Administration (NASA), the Netherlands Agency for Aerospace Programs (NIVR), and the U.K. Science and Engineering Research Council (SERC).

†Operated by AURA, Inc., under contract with the National Science Foundation.

\section{Introduction}

The Infrared Astronomical Satellite(IRAS) performed a survey that covered $96 \%$ of the sky and is complete to $0.5 \mathrm{Jy}$ at wavelengths of $12 \mu \mathrm{m}, 25 \mu \mathrm{m}$, and $60 \mu \mathrm{m}$ and to $1.5 \mathrm{Jy}$ at $100 \mu \mathrm{m}$. The survey sensitivity at $12 \mu \mathrm{m}$ corre- 
sponds to about $[12 \mu \mathrm{m}]=4.4 \mathrm{mag}$. Previous surveys of the infrared sky include The Revised AFGL Infrared Sky Survey Catalog (Price and Murdock 1983; henceforth RAFGL) which covered $92.5 \%$ of the sky at $11 \mu \mathrm{m}$ and was complete to about $[11 \mu \mathrm{m}]=1.0 \mathrm{mag}$ and the Caltech Two Micron Sky Survey Catalog (Neugebauer and Leighton 1969; henceforth IRC) which was complete for declinations north of $-33^{\circ}$ to $[2.2 \mu \mathrm{m}]<3.0 \mathrm{mag}$.

In this paper, the brightest high-galactic-latitude 12 $\mu \mathrm{m}$ point sources with $[12 \mu \mathrm{m}]<0$ mag found by IRAS have been selected with the intention of assembling the data for sources which can be studied easily from the ground. No detailed discussion of the physical properties of these sources is given. No new population of astrophysical objects is found among this sample of IRAS sources.

\section{Observations}

The IRAS Point Source Catalog (1984) was searched for $12 \mu \mathrm{m}$ sources with an average $12 \mu \mathrm{m}$ flux density greater than $28 \mathrm{Jy}(0 \mathrm{mag})$ and an absolute galactic latitude greater than $30^{\circ}$. The Large Magellanic Cloud was omitted due to the high density of sources there. These selection criteria improve the reliability of the $60 \mu \mathrm{m}$ and $100 \mu \mathrm{m}$ measurements that tend to be most affected by the "cirrus" (Low et al. 1984) and the high source density found at lower latitudes.

The search of the catalog resulted in 269 sources. In addition, two sources not found in the Point Source Cata$\log (22042+1138$ and $22596+1019)$ are included that lie within a narrow region (about $2 \%$ of the sky) observed by one or more scans of the satellite but not enough times to be properly confirmed (IRAS Explanatory Supplement 1984; henceforth the Supplement). The IRAS data for these sources are given in Table I. The source name is given in the same format as used in the IRAS Point Source Catalog. The flux densities were color-corrected by fitting flux ratios of neighboring wavelength bands to a Planck function. [If a wavelength band was observed fluxes in two neighboring bands, then an average flux density was used from the two fits.] When a $100 \mu \mathrm{m}$ upper limit is quoted the $60 \mu \mathrm{m}$ flux density was color corrected using only the $25 \mu \mathrm{m}-60 \mu \mathrm{m}$ fit. All flux densities discussed in this paper, including the $28 \mathrm{Jy}$ threshold at $12 \mu \mathrm{m}$, refer to the color-corrected values.

Sources with flux densities brighter than $28 \mathrm{Jy}$ at $12 \mu \mathrm{m}$ are approximately 50 times brighter than the completeness limit estimated in the Supplement. Thus, the present sample ought to be $100 \%$ complete in the surveyed area, as well as $100 \%$ reliable (no false sources). Excluding incompleteness due to variability we conclude that the completeness of the sample is simply the completeness of the sky coverage, i.e., $93 \%$ of the sky with galactic latitude greater than $30^{\circ}$.

The relative photometric precision for detected sources is wavelength dependent and ranges from $5 \%$ to $10 \%(1 \sigma)$ for the $12,25,60$, and the bright $(>3 \mathrm{Jy}) 100 \mu \mathrm{m}$ detections. Flux densities greater than $100 \mathrm{Jy}$ at $100 \mu \mathrm{m}$ may have larger uncertainties due to detector nonlinearities. The fainter $100 \mu \mathrm{m}$ detections have relative photometric uncertainties that range from $10 \%$ to $50 \%$. The absolute calibration is accurate to $5 \%$ at 12,25 , and $60 \mu \mathrm{m}$ and $10 \%$ at $100 \mu \mathrm{m}$ (Supplement).

The positional uncertainty of the sample was determined from the 176 sources that have optical counterparts listed in the Smithsonian Astrophysical Observatory Star Catalog (SAO 1966; henceforth SAO). No statistically significant mean positional discrepancy was found. The rms dispersion about the mean was found to be 1.9 along the IRAS scan direction (Gaussian distribution) and 6".9 perpendicular to the IRAS scan direction (non-Gaussian distribution, Fowler and Rolfe (1982)). Since there are no systematic differences between the sources found in the SAO and those that are not, these positional uncertainties should apply to the sample as a whole. These positional uncertainties are typical of those quoted in the Point Source Catalog for sources detected at $12 \mu \mathrm{m}$ and $25 \mu \mathrm{m}$.

For 30 of the stars, the Point Source Catalog indicated the existence of nearby small extended sources at various wavelengths. Because of the rudimentary nature of the processing of the small extended sources, it is necessary to examine the reality of the extended structure more carefully. In fact, as discussed below, only 12 of the 30 sources showed true extended emission at $60 \mu \mathrm{m}$ or 100 $\mu \mathrm{m}$. No source showed extended emission at $12 \mu \mathrm{m}$ or 25 $\mu \mathrm{m}$.

Fifteen of the 30 small extended sources were caused by optical cross-talk from the bright point source. The total flux density was a few percent to one-third of the flux density in the bright point sources. The point-source flux densities for these 15 stars were adopted.

For the remaining 15 stars, the detector output for all survey scans was retrieved and the scans over each source measured independently for extent and total emission. One source was found to be pointlike with some evidence for a faint asymmetric extension. Two sources had nearby point-source companions; the combination was detected as an extended source on some scans. For those three sources, the point-source flux densities listed in the catalog were kept.

For eight of the remaining 12 sources, total flux densities at $60 \mu \mathrm{m}$ and $100 \mu \mathrm{m}$ were estimated by integrating the output from each detector that scanned over the source. For the last four, the source extent at $60 \mu \mathrm{m}$ and $100 \mu \mathrm{m}$ in the scan direction was larger than a detector length (about $5^{\prime}$ ) so the total flux estimate involved adding the signals from pairs of adjacent detectors. Thus a total of 12 sources have had their point-source flux densities corrected to take into account extended emission and are 
TABLE I

Cataloged Parameters

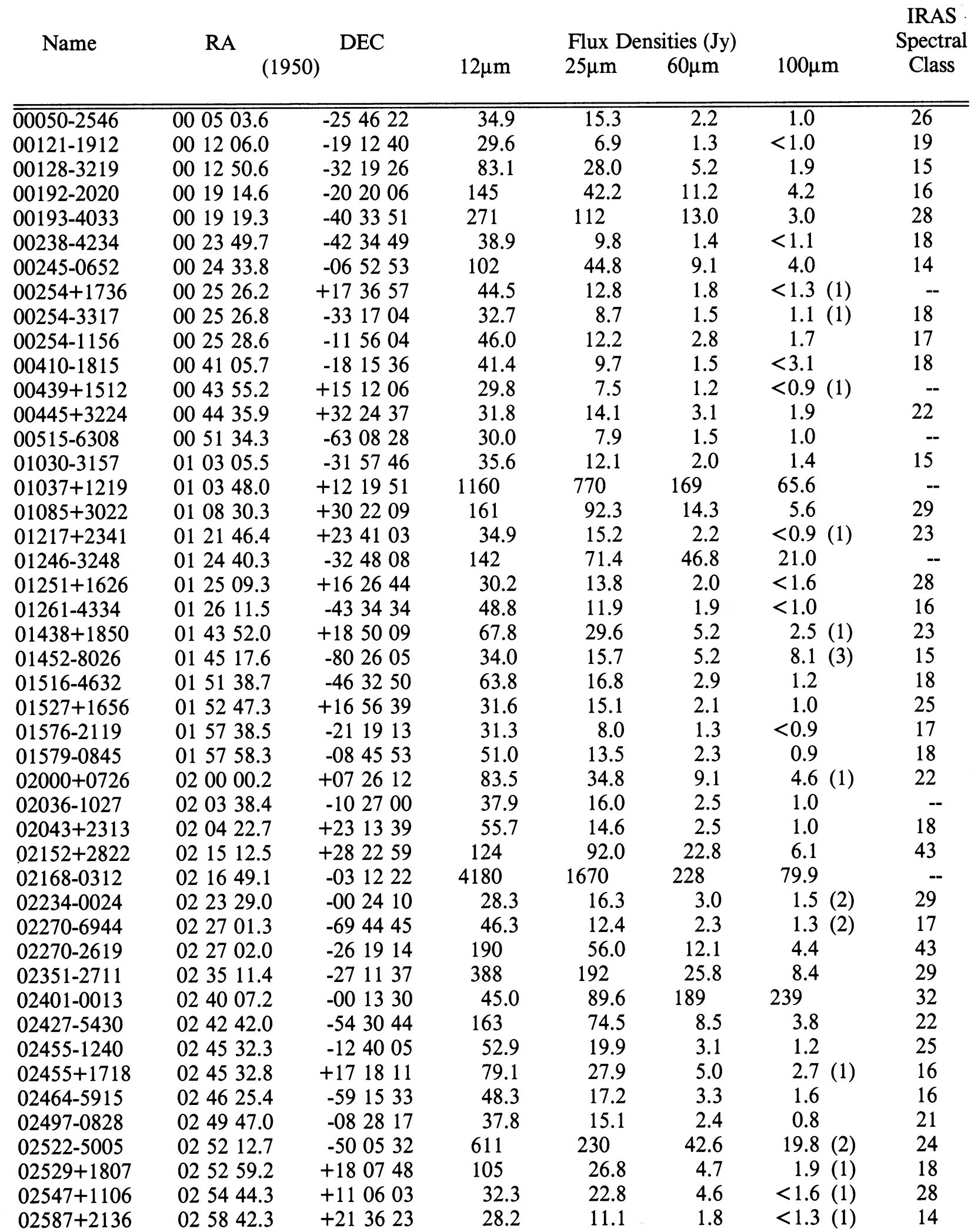


TABLE I (Continued)

\begin{tabular}{|c|c|c|c|c|c|c|c|}
\hline \multirow[t]{2}{*}{ Name } & \multirow[t]{2}{*}{ RA } & \multirow[t]{2}{*}{ DEC } & \multicolumn{4}{|c|}{ Flux Densities (Jy) } & \multirow{2}{*}{$\begin{array}{c}\text { IRAS } \\
\text { Spectral } \\
\text { Class }\end{array}$} \\
\hline & & & $12 \mu \mathrm{m}$ & $25 \mu \mathrm{m}$ & $60 \mu \mathrm{m}$ & $100 \mu \mathrm{m}$ & \\
\hline $02596+0353$ & 025939.8 & " +035338 & 164 & 39.6 & 6.6 & $2.1(1)$ & 18 \\
\hline $03082+1436$ & 030816.2 & +143640 & 56.8 & 19.4 & 3.8 & $1.5(1)$ & 21 \\
\hline $03112-5730$ & 031116.8 & -573026 & 83.1 & 29.7 & 5.6 & 2.3 & 43 \\
\hline $03172-2156$ & $\begin{array}{lll}03 & 17 & 17.3\end{array}$ & -215621 & 113 & 26.9 & 4.3 & 1.4 & 17 \\
\hline 03287-1535 & 032844.8 & -153502 & 54.4 & 41.4 & 9.8 & $3.4(1)$ & 29 \\
\hline 03318-1619 & 033153.6 & -161948 & 129 & 45.3 & 7.5 & $3.2(1)$ & 15 \\
\hline 03336-7636 & 033340.0 & -763657 & 41.9 & 17.1 & 2.7 & $1.0(1,2)$ & 26 \\
\hline 03364-5533 & 033629.4 & -553330 & 57.6 & 21.3 & 3.0 & $1.3(1)$ & 16 \\
\hline 03463-0710 & 034620.6 & -071001 & 28.5 & 10.0 & 3.9 & $7.9(1,3)$ & 17 \\
\hline $03479-7423$ & 034759.7 & -742329 & 79.1 & 20.2 & 3.1 & $1.2(1)$ & 18 \\
\hline $03482-5213$ & 034813.9 & -521349 & 51.1 & 20.7 & 2.8 & 1.2 & 22 \\
\hline 03489-0131 & 034854.8 & -013114 & 69.2 & 28.0 & 4.6 & $2.5(1)$ & 24 \\
\hline 03505-0919 & 035030.6 & -091901 & 33.7 & 12.5 & 2.0 & $<1.0(1)$ & 26 \\
\hline $03507+1115$ & 035043.5 & +111529 & 4080 & 1770 & 252 & $93.0(1)$ & 26 \\
\hline $03511-4558$ & 035111.8 & -455838 & 49.0 & 19.5 & 2.8 & 1.3 & 25 \\
\hline 03557-1339 & 035542.1 & -133900 & 77.4 & 19.3 & 3.5 & 1.0 & 18 \\
\hline 04001-6217 & 040010.0 & $-62 \quad 1751$ & 59.3 & 15.7 & 2.4 & 0.8 & 18 \\
\hline $04020-1551$ & 040201.6 & -155138 & 295 & 137 & 19.7 & $8.9(3)$ & 22 \\
\hline 04094-2515 & 040925.2 & -251544 & 82.0 & 31.1 & 4.6 & $2.0(1)$ & 24 \\
\hline $04140-8158$ & 041400.8 & -815853 & 292 & 118 & 18.6 & $7.4(2)$ & 24 \\
\hline $04157-1837$ & 041542.5 & -183742 & 35.2 & 12.8 & 2.1 & 1.2 & -- \\
\hline 04311-0004 & 043111.3 & -000436 & 33.3 & 12.6 & 2.0 & $1.0(1,2)$ & 14 \\
\hline 04330-6307 & 043301.7 & -630745 & 29.3 & 9.8 & 1.6 & $1.2(1)$ & 15 \\
\hline $04345-2740$ & 043433.4 & -274044 & 29.6 & 11.7 & 2.1 & 1.2 & 15 \\
\hline $04361-6210$ & 043610.4 & $\begin{array}{llll}-62 & 10 & 27\end{array}$ & 4240 & 1268 & 189 & 73.6 & -- \\
\hline $04382-1417$ & 043814.8 & -141747 & 92.1 & 39.7 & 6.8 & $3.6(1)$ & 21 \\
\hline 04382-1946 & 043815.1 & -194602 & 48.0 & 12.2 & 2.2 & 1.0 & 18 \\
\hline 04387-3819 & 043846.0 & $\begin{array}{llll}-38 & 19 & 49\end{array}$ & 132 & 49.5 & 7.2 & 2.9 & 23 \\
\hline 04404-7427 & 044026.9 & -742727 & 63.4 & 37.2 & 5.2 & $1.9(2)$ & 29 \\
\hline 04573-1452 & 045719.4 & -145249 & 287 & 87.4 & 20.4 & $8.2(1,2)$ & 45 \\
\hline $05027-2158$ & 050242.8 & -215820 & 139 & 61.0 & 9.3 & $4.8(2)$ & 15 \\
\hline 05033-2226 & 050321.0 & -222618 & 41.5 & 11.3 & 1.6 & $<1.0$ & 18 \\
\hline 05069-3434 & 050658.3 & -343447 & 80.5 & 28.3 & 6.4 & 3.4 & 16 \\
\hline $05071-6327$ & $0507 \quad 10.1$ & -632744 & 29.5 & 7.4 & 1.2 & $<0.9$ & 18 \\
\hline 05096-4834 & 050937.6 & -483401 & 168 & 66.7 & 11.5 & $5.1(2)$ & 25 \\
\hline 05098-6422 & 050950.5 & -642241 & 120 & 62.2 & 12.8 & $5.9(2)$ & 26 \\
\hline $05217-3943$ & 052144.8 & -394323 & 29.6 & 14.7 & 2.3 & $<1.3$ & 26 \\
\hline $05418-4628$ & 054150.7 & -462830 & 42.2 & 13.1 & 4.5 & 3.5 & 41 \\
\hline $08063+6522$ & 080618.5 & +652221 & 40.1 & 16.6 & 3.2 & 1.7 & 16 \\
\hline $08525+1725$ & 085234.2 & +172522 & 66.2 & 19.5 & 5.8 & 3.0 & 42 \\
\hline $08538+2002$ & 085348.8 & +200230 & 44.4 & 12.3 & 3.0 & 1.3 & 23 \\
\hline $08555+1102$ & 085533.1 & +110222 & 60.1 & 21.3 & 3.2 & 1.2 & 41 \\
\hline $.09057+1325$ & 090542.1 & +132523 & 78.6 & 33.7 & 6.5 & 3.7 & 15 \\
\hline $09069+2527$ & 090657.9 & +252706 & 56.5 & 24.4 & 4.4 & 1.9 & 23 \\
\hline $09076+3110$ & 090737.7 & +311004 & 404 & 154 & 24.5 & 8.9 & 22 \\
\hline $09180+3436$ & 091800.7 & +343617 & 60.0 & 13.9 & 2.3 & 0.9 & 18 \\
\hline $09180+5654$ & 091804.4 & +565443 & 29.1 & 7.8 & 1.3 & $<1.0$ & 18 \\
\hline
\end{tabular}


TABLE I (Continued)

\begin{tabular}{llllcrcc} 
Name & RA & DEC & \multicolumn{3}{c}{ IRAS } \\
& & (1950) & & Flux Densities (Jy) & Spectral \\
& & $12 \mu \mathrm{m}$ & $25 \mu \mathrm{m}$ & $60 \mu \mathrm{m}$ & $100 \mu \mathrm{m}$ & Class
\end{tabular}

\begin{tabular}{|c|c|c|c|c|c|c|c|}
\hline$\overline{09425+3444}$ & 094234.4 & +344435 & 354 & 129 & 19.5 & 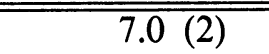 & $\overline{24}$ \\
\hline $09429+5721$ & 094300.0 & +572133 & 28.8 & 7.4 & 1.3 & $<1.0$ & 17 \\
\hline $09448+1139$ & 094452.1 & +113941 & 1625 & 475 & 88.7 & $35.6(2)$ & -- \\
\hline $09452+1330$ & $0945 \quad 14.2$ & +133040 & 41207 & 18000 & 4375 & 846 & 43 \\
\hline $09517+6954$ & 095142.4 & +695459 & 64.1 & 300 & 1200 & $1130(1)$ & 81 \\
\hline $10131+3049$ & 101310.7 & +304917 & 2660 & 927 & 212 & 77.9 & 04 \\
\hline $10172+2005$ & 101713.6 & +200538 & 59.2 & 14.2 & 2.7 & 1.0 & 18 \\
\hline $10193+4145$ & 101919.5 & +414513 & 71.5 & 18.1 & 3.0 & 1.3 & -- \\
\hline $10305+7001$ & 103035.0 & +700123 & 28.0 & 11.1 & 1.9 & 1.0 & 22 \\
\hline $10350-1307$ & 103503.2 & -130716 & 163 & 55.2 & 45.0 & 40.2 (3) & -- \\
\hline $10353-1145$ & 103520.5 & -114522 & 61.1 & 29.1 & 4.7 & 1.9-- & \\
\hline $10411+6902$ & 104107.3 & +690218 & 85.7 & 40.9 & 5.5 & $2.5(2)$ & 23 \\
\hline $10416+6740$ & 104138.1 & +674021 & 37.5 & 11.0 & 6.8 & $5.8(3)$ & 42 \\
\hline 10491-2059 & 104911.4 & -205906 & 921 & 349 & 75.8 & 26.7 & -- \\
\hline $10521+7208$ & 105207.6 & +720812 & 32.3 & 15.6 & 3.4 & $1.5(1,2)$ & 26 \\
\hline $10580-1803$ & 105805.6 & -180320 & 552 & 227 & 39.1 & 17.9 & 22 \\
\hline $11006+6201$ & 110038.9 & +620114 & 59.5 & 14.3 & 2.4 & 1.0 & 18 \\
\hline $11125+7524$ & 111232.1 & +752454 & 82.0 & 37.2 & 5.7 & 3.1 & 23 \\
\hline $11251+4527$ & 112506.6 & +452739 & 36.9 & 13.9 & 2.5 & 1.1 & -- \\
\hline $11252+1525$ & 112516.4 & +152522 & 44.8 & 23.0 & 2.9 & 1.2 & -- \\
\hline $11284+6936$ & 112827.8 & +693621 & 38.7 & 10.0 & 1.7 & $<1.0$ & 18 \\
\hline $11308-1020$ & 113052.4 & -102026 & 46.7 & 16.7 & 3.7 & 1.2 & 44 \\
\hline $11358+0824$ & 113553.3 & +082439 & 44.2 & 11.3 & 2.1 & $<1.2$ (1) & 18 \\
\hline $11432+0648$ & 114316.6 & +064835 & 30.5 & 7.8 & 1.3 & $<1.0$ & -- \\
\hline $11445+4344$ & 114436.0 & +434457 & 72.4 & 40.2 & 5.2 & 2.2 & -- \\
\hline $11462-2628$ & 114613.0 & $\begin{array}{ll}-26 & 2817\end{array}$ & 47.4 & 12.2 & 2.3 & 0.9 & 18 \\
\hline $11473-2718$ & 114719.4 & $\begin{array}{lll}-27 & 18 & 17\end{array}$ & 32.3 & 12.5 & 1.9 & $<1.3$ & 22 \\
\hline $11485-1055$ & 114832.8 & -105547 & 66.4 & 23.0 & 4.8 & 4.2 & -- \\
\hline 11501-0719 & 115011.7 & -071908 & 44.7 & 16.8 & 3.3 & 1.7 & 16 \\
\hline $11538+5808$ & 115354.0 & +580859 & 53.3 & 19.1 & 3.3 & 1.5 & 16 \\
\hline $12020+0254$ & 120201.6 & +25400 & 28.1 & 13.7 & 2.3 & $<1.2$ & 26 \\
\hline $12046-0629$ & 120441.2 & -062916 & 72.1 & 26.5 & 3.6 & $1.6(1)$ & -- \\
\hline $12075-2220$ & 120732.1 & -222025 & 28.9 & 7.3 & 1.4 & $<1.2$ (1) & 17 \\
\hline $12226+0102$ & 122240.5 & +010247 & 69.8 & 21.2 & 4.3 & 1.6 & 04 \\
\hline $12277+0441$ & 122747.8 & +044134 & 207 & 76.0 & 15.0 & 6.9 & 15 \\
\hline $12344+2720$ & 123424.4 & +272030 & 66.4 & 28.4 & 4.1 & 2.1 & -- \\
\hline $12345-1715$ & 123430.0 & -171504 & 28.9 & 11.4 & 3.4 & 3.1 & -- \\
\hline $12380+5607$ & 123803.5 & +560719 & 166 & 67.9 & 12.9 & 6.8 & 15 \\
\hline $12427+4542$ & 124247.2 & +454245 & 197 & 52.8 & 23.3 & $9.6(3)$ & 42 \\
\hline $12447+0425$ & 124445.8 & +042503 & 173 & 51.1 & 10.3 & 3.4 & 44 \\
\hline $12517-0915$ & 125144.3 & -091559 & 32.5 & 7.5 & 1.4 & $<2.1$ & 18 \\
\hline $12526+4728$ & 125239.7 & +472802 & 41.2 & 10.9 & 1.8 & $<1.0$ & -- \\
\hline $12530+0340$ & 125304.4 & +034003 & 115 & 28.8 & 4.4 & 1.5 & 18 \\
\hline $12544+6615$ & 125427.1 & +661557 & 80.0 & 23.7 & 6.7 & 3.9 & 41 \\
\hline $12562+2324$ & 125612.1 & +232434 & 30.9 & 15.0 & 2.8 & 1.1 & 28 \\
\hline
\end{tabular}


TABLE I (Continued)

\begin{tabular}{|c|c|c|c|c|c|c|c|}
\hline Name & RA & DEC & $12 \mu \mathrm{m}$ & \multicolumn{2}{|c|}{ Flux Densities (Jy) } & $100 \mu \mathrm{m}$ & $\begin{array}{c}\text { IRAS } \\
\text { Spectral } \\
\text { Class }\end{array}$ \\
\hline $13001+0527$ & 130006.1 & +052714 & 401 & $\overline{168}$ & $\overline{\overline{30.7}}$ & $\overline{13.4}$ & 21 \\
\hline $13039+2253$ & 130356.4 & +225303 & 46.0 & 12.1 & 2.3 & 1.2 & 18 \\
\hline $13114-0232$ & 131129.7 & -023234 & 595 & 252 & 37.2 & 13.5 & -- \\
\hline $13172+4547$ & $1317 \quad 17.1$ & +454720 & 114 & 47.4 & 4.1 & $1.3(1)$ & 29 \\
\hline 13269-2301 & 132658.4 & -230127 & 1280 & 427 & 85.6 & 30.9 (3) & 15 \\
\hline 13303-0656 & 133022.8 & -065618 & 112 & 40.2 & 6.1 & 2.0 & 16 \\
\hline 13462-2807 & 134612.5 & $-2807 \quad 11$ & 3086 & 855 & 153 & 66.0 & 02 \\
\hline $13468+3947$ & 134648.1 & +394728 & 84.4 & 29.7 & 4.6 & 1.7 & 15 \\
\hline $13492-0325$ & 134915.5 & -032543 & 40.1 & 16.3 & 2.9 & 1.0 & 16 \\
\hline $13495+3441$ & 134934.8 & +344130 & 33.9 & 8.1 & 1.3 & $<1.1$ & 80 \\
\hline $13499+6458$ & 134957.2 & +645815 & 39.9 & 10.6 & 1.9 & 0.9 & 18 \\
\hline $13582+3806$ & 135814.6 & +380643 & 39.9 & 20.6 & 3.6 & 1.4 & -- \\
\hline $14059+4405$ & 140555.5 & +440530 & 50.0 & 13.4 & 2.2 & $<1.1$ & 18 \\
\hline 14086-0730 & 140838.9 & -073044 & 136 & 77.7 & 12.8 & 4.3 & -- \\
\hline 14086-2839 & 140840.9 & -283903 & 67.8 & 29.1 & 5.3 & 2.1 & 26 \\
\hline $14133+1925$ & 141319.6 & +192527 & 547 & 116 & 19.3 & 7.0 & 18 \\
\hline $14142-1612$ & 141414.6 & -161229 & 56.9 & 25.5 & 4.4 & 1.8 & 22 \\
\hline $14162+6701$ & 141613.4 & +670128 & 51.2 & 18.4 & 2.7 & $1.2(1)$ & 15 \\
\hline $14219+2555$ & 142156.4 & +255548 & 737 & 310 & 53.9 & 23.3 & -- \\
\hline $14247+0454$ & 142445.2 & +045407 & 100 & 49.5 & 9.2 & 3.8 & 24 \\
\hline $14277+3904$ & 142743.8 & +390500 & 32.9 & 10.5 & 1.6 & $<1.0$ & 16 \\
\hline $14371+3245$ & 143709.0 & +324515 & 113 & 52.4 & 8.2 & 3.1 & 22 \\
\hline $14390+3147$ & 143905.9 & +314707 & 53.1 & 22.5 & 4.5 & 2.6 & 23 \\
\hline $14412+2644$ & 144113.9 & +264419 & 28.1 & 7.3 & 1.2 & $<1.0$ & 18 \\
\hline $14427+2717$ & 144247.9 & +271704 & 32.0 & 7.6 & 1.1 & $<1.0$ & -- \\
\hline $14437+1520$ & 144344.3 & +152026 & 77.4 & 21.1 & 4.0 & $1.6(1)$ & 17 \\
\hline $14508+7421$ & 145048.9 & +742145 & 112 & 27.0 & 4.4 & 1.8 & 18 \\
\hline $14550-1214$ & 145500.9 & -121408 & 78.3 & 31.4 & 5.5 & 2.7 & .22 \\
\hline $14567+6607$ & 145646.0 & +660757 & 89.5 & 23.7 & 3.9 & 1.1 & 18 \\
\hline $15060+0947$ & 150600.2 & +094743 & 34.7 & 20.4 & 3.0 & 1.2 & 28 \\
\hline $15193+1429$ & $\begin{array}{lll}15 & 19 & 19.4\end{array}$ & +142933 & 35.9 & 14.5 & 5.0 & 4.2 & 22 \\
\hline $15193+3132$ & $\begin{array}{lll}15 & 19 & 20.5\end{array}$ & +313247 & 187 & 94.3 & 14.9 & 6.4 & 24 \\
\hline $15223-0203$ & $\begin{array}{lll}15 & 22 & 19.0\end{array}$ & -020334 & 95.0 & 49.9 & 8.6 & 3.1 & 24 \\
\hline $15255+1944$ & 152532.2 & +194410 & 220 & 113 & 13.8 & $4.7(1)$ & 29 \\
\hline $15262+0400$ & 152613.6 & +040002 & 43.9 & 23.3 & 3.5 & 1.3 & 27 \\
\hline $15298+0348$ & 152953.8 & +034836 & 37.3 & 14.1 & 1.8 & $<1.1(1)$ & 14 \\
\hline $15314+7847$ & 153124.1 & +784754 & 120 & 43.2 & 5.7 & $2.3(2)$ & 21 \\
\hline $15341+1515$ & 153408.8 & +151555 & 163 & 68.2 & 12.5 & 5.0 & 22 \\
\hline $15361+2441$ & 153607.5 & +244105 & 64.6 & 17.8 & 4.6 & $2.7(3)$ & 17 \\
\hline $15410-0133$ & 154100.4 & -013309 & 98.1 & 34.7 & 5.6 & 2.2 & 21 \\
\hline $15418+0634$ & 154149.2 & +063453 & 28.7 & 7.1 & 1.2 & $<1.0(1)$ & 18 \\
\hline $15464+1817$ & 154629.1 & +181737 & 31.8 & 8.4 & 1.7 & $1.2(1)$ & 18 \\
\hline $15465+2818$ & 154631.7 & +281829 & 32.9 & 13.2 & 6.1 & $8.3(3)$ & 15 \\
\hline $15477+3943$ & 154744.9 & +394315 & 79.2 & 23.8 & 4.8 & 1.8 & -- \\
\hline $15483+1517$ & 154823.0 & +151702 & 154 & 51.9 & 8.5 & 3.2 & 24 \\
\hline $15492+4837$ & 154916.0 & +483755 & 173 & 72.2 & 12.8 & 5.3 & 41 \\
\hline $15566+3609$ & 155638.9 & +360948 & 46.8 & 20.7 & 2.6 & 1.2 & 26 \\
\hline
\end{tabular}


TABLE I (Continued)

\begin{tabular}{|c|c|c|c|c|c|c|c|}
\hline Name & RA & DEC & \multicolumn{4}{|c|}{ Flux Densities (Jy) } & $\begin{array}{l}\text { IRAS } \\
\text { Spectral } \\
\text { Class }\end{array}$ \\
\hline $16011+4722$ & 160107.9 & +472236 & 423 & 179.0 & 31.7 & $\overline{\overline{16.7}}$ & 24 \\
\hline $16081+2511$ & 160808.6 & +251159 & 147 & 57.7 & 8.9 & 4.0 & 23 \\
\hline $16095+2337$ & 160930.0 & +233722 & 32.1 & 8.3 & 1.4 & $<1.0(1)$ & 17 \\
\hline $16117-0334$ & 161143.1 & -033406 & 106 & 26.7 & 4.4 & $1.6(1)$ & 18 \\
\hline $16164+5952$ & 161624.7 & +595232 & 34.9 & 9.4 & 1.8 & 1.1 & 18 \\
\hline $16235+1900$ & 162334.8 & +190015 & 398 & 131 & 20.9 & $8.7(2)$ & 23 \\
\hline $16260+3454$ & 162600.6 & +345445 & 61.9 & 38.9 & 8.0 & $3.3(2)$ & 28 \\
\hline $16269+4159$ & 162700.0 & +415923 & 343 & 108 & 17.7 & 5.8 & 16 \\
\hline $16306+7223$ & 163037.2 & +722314 & 88.6 & 33.2 & 5.7 & 3.1 & 15 \\
\hline $16418+5459$ & 164151.7 & +545945 & 118 & 54.5 & 9.4 & 4.0 & 24 \\
\hline $16432+1213$ & 164313.9 & +121337 & 28.8 & 9.7 & 1.5 & $<1.0(1,2)$ & 15 \\
\hline $16473+5753$ & 164723.8 & +575358 & 51.0 & 17.4 & 2.9 & 1.4 & 22 \\
\hline $17081+6422$ & 170806.2 & +642253 & 50.9 & 19.5 & 3.8 & 2.1 & 15 \\
\hline $17086+4045$ & 170840.4 & +404501 & 29.0 & 10.0 & 2.5 & 1.5 & 17 \\
\hline $17133+3651$ & 171318.0 & +365151 & 33.6 & 8.1 & 1.3 & $<1.0(1)$ & 18 \\
\hline $17190+2658$ & 171904.3 & +265842 & 29.0 & 12.1 & 2.1 & $1.2(1)$ & 26 \\
\hline $17329+5359$ & 173255.0 & +535931 & 32.3 & 16.4 & 3.3 & $1.4(2)$ & 25 \\
\hline $17361+5746$ & 173611.7 & +574606 & 63.6 & 34.6 & 5.8 & 2.4 & 28 \\
\hline $19510-5919$ & 195101.4 & -591938 & 415 & 149 & 22.5 & 9.4 & 14 \\
\hline $19575-5930$ & 195733.3 & -593053 & 164 & 42.1 & 7.5 & 2.7 & 18 \\
\hline $20042-4241$ & 200415.7 & -424105 & 214 & 120 & 21.2 & $8.0(1,2)$ & 28 \\
\hline $20075-6005$ & 200733.4 & -600512 & 484 & 205 & 40.3 & 17.6 & 22 \\
\hline $20111-4708$ & 201110.8 & -470807 & 35.0 & 12.6 & 2.4 & $1.4(2)$ & 15 \\
\hline $20120-4433$ & 201200.2 & -443348 & 36.1 & 21.0 & 16.9 & $26.7(2,3)$ & 16 \\
\hline $20135-7152$ & 201335.6 & -715253 & 118 & 70.9 & 12.1 & $4.3(2)$ & 29 \\
\hline 20144-3916 & 201425.4 & -391606 & 33.1 & 10.8 & 1.7 & $<1.2$ & 16 \\
\hline $20165-5051$ & 201633.2 & -505141 & 55.0 & 22.2 & 4.3 & 2.5 & 16 \\
\hline $20248-2825$ & 202451.9 & -282541 & 403 & 141 & 25.5 & 11.5 & 15 \\
\hline $20259-4035$ & 202556.5 & -403501 & 29.1 & 13.4 & 3.1 & $1.4(2)$ & 24 \\
\hline 20296-2151 & 202938.7 & -215140 & 30.3 & 15.7 & 2.4 & $<1.0(1,2)$ & 28 \\
\hline 20359-3806 & 203556.8 & -380627 & 71.9 & 29.2 & 5.4 & 2.5 & 21 \\
\hline 20484-7202 & 204829.3 & -720248 & 166 & 82.2 & 10.5 & $3.8(2)$ & 29 \\
\hline $20526-5431$ & 205241.2 & -543100 & 53.4 & 25.6 & 4.0 & $1.8(2)$ & 22 \\
\hline $20541-6549$ & 205407.9 & -654945 & 157 & 66.0 & 11.2 & $4.2(2)$ & 29 \\
\hline 21044-1637 & 210428.8 & -163723 & 120 & 83.9 & 13.5 & 5.8 & 27 \\
\hline $21069-3843$ & 210657.0 & -384318 & 163 & 87.3 & 12.1 & $3.8(2)$ & 29 \\
\hline $21100-1435$ & 211001.2 & -143555 & 53.3 & 20.4 & 3.0 & $<1.0(1)$ & 24 \\
\hline $21168-4514$ & 211649.7 & -451412 & 34.6 & 10.5 & 4.1 & 2.8 & 17 \\
\hline $21197-6956$ & 211946.9 & -695655 & 58.4 & 20.8 & 9.1 & $6.0(3)$ & 22 \\
\hline $21206-4054$ & 212038.1 & -405459 & 113 & 58.0 & 6.5 & $2.8(2)$ & 29 \\
\hline $21243-6943$ & 212419.0 & -694326 & 80.1 & 21.1 & 3.8 & 1.5 & 18 \\
\hline $21321+0136$ & 213210.0 & +013620 & 29.4 & 8.2 & 1.4 & $<1.0(1)$ & 18 \\
\hline $21368-3812$ & 213649.5 & $-38 \quad 1252$ & 51.1 & 25.8 & 5.7 & 3.1 & 21 \\
\hline $21377-0200$ & 213744.7 & -020048 & 54.8 & 25.0 & 4.3 & 2.2 & 14 \\
\hline $21417+0938$ & 214144.1 & +093844 & 72.9 & 17.9 & 3.0 & $<1.2(1)$ & 18 \\
\hline
\end{tabular}


TABLE I (Continued)

\begin{tabular}{|c|c|c|c|c|c|c|c|}
\hline \multirow[t]{2}{*}{ Name } & \multirow[t]{2}{*}{ RA } & \multirow[t]{2}{*}{ DEC } & \multicolumn{4}{|c|}{ Flux Densities (Jy) } & \multirow{2}{*}{$\begin{array}{c}\text { IRAS } \\
\text { Spectral } \\
\text { Class }\end{array}$} \\
\hline & & & $12 \mu \mathrm{m}$ & $25 \mu \mathrm{m}$ & $60 \mu \mathrm{m}$ & $100 \mu \mathrm{m}$ & \\
\hline$\overline{21439-0226}$ & 214356.8 & -022639 & 558 & 238 & 36.2 & $\overline{14.7}$ & -- \\
\hline $21453-4708$ & 214519.3 & -470845 & 29.9 & 11.1 & 1.7 & $0.9(2)$ & 15 \\
\hline $21543-1421$ & 215419.6 & -142104 & 70.2 & 35.9 & 6.3 & 2.3 & 21 \\
\hline $22048+1138$ & 220449.0 & +113834 & 162 & 80.9 & 12.4 & $3.5(4)$ & -- \\
\hline $22142-8454$ & $2214 \quad 14.7$ & -845459 & 125 & 53.4 & 8.5 & 3.9 & 23 \\
\hline $22145-8041$ & 221433.3 & -804126 & 107 & 28.2 & 6.5 & $3.3(1,3)$ & -- \\
\hline $22150-6030$ & 221505.0 & -603038 & 41.8 & 10.3 & 1.6 & $<1.2$ & 18 \\
\hline $22190-0751$ & 221904.0 & -075141 & 72.9 & 33.0 & 5.3 & 2.8 & 25 \\
\hline $22196-4612$ & 221940.8 & -461207 & 785 & 326 & 58.4 & 20.7 & 42 \\
\hline $22230-4841$ & 222300.6 & -484137 & 109 & 42.5 & 6.7 & $2.9(2)$ & 22 \\
\hline $22231-4529$ & 222309.4 & -452930 & 104 & 48.4 & 6.0 & $1.9(2)$ & 29 \\
\hline $22267-4400$ & 222646.8 & -440020 & 78.1 & 19.9 & 3.5 & 1.2 & 18 \\
\hline $22280+1250$ & 222800.3 & +125053 & 31.1 & 10.9 & 2.1 & $1.5(1)$ & 15 \\
\hline $22296-6214$ & 222939.0 & -621422 & 42.7 & 10.8 & 1.9 & $<1.0$ & 18 \\
\hline $22359-1417$ & 223555.8 & -141747 & 34.7 & 15.1 & 2.6 & 1.1 & 15 \\
\hline $22396-4708$ & 223941.8 & -470849 & 667 & 170 & 30.6 & 10.6 & -- \\
\hline $22516+0838$ & 225141.1 & +083812 & 103 & 48.2 & 8.8 & 3.5 & 29 \\
\hline $22525-2952$ & 225234.9 & -295247 & 209 & 81.3 & 13.8 & 5.3 & 22 \\
\hline $22540-5740$ & 225403.3 & -574003 & 106 & 41.4 & 7.4 & 4.1 & 22 \\
\hline $22596+1019$ & 225936.7 & +101917 & 112 & 65.3 & 9.9 & $3.3(4)$ & -- \\
\hline $23041+1016$ & 230408.8 & +101625 & 151 & 53.7 & 8.5 & 3.5 & 22 \\
\hline $23063-3024$ & 230623.5 & -302418 & 93.4 & 34.6 & 4.7 & 2.1 & -- \\
\hline $23070+0824$ & 230701.6 & +082432 & 57.2 & 14.4 & 2.5 & $1.0(1)$ & 18 \\
\hline $23086+0443$ & 230841.8 & +044359 & 48.0 & 13.6 & 2.7 & 1.6 & 17 \\
\hline 23134-7031 & 231326.6 & -703131 & 84.9 & 27.3 & 4.5 & 2.0 & 16 \\
\hline $23142-0759$ & 231415.3 & -080000 & 46.3 & 12.1 & 2.0 & $<1.0$ & -- \\
\hline $23142+1019$ & 231417.0 & +101938 & 28.9 & 8.0 & 2.0 & $0.9(1)$ & 17 \\
\hline $23166+1655$ & 231641.7 & +165503 & 749 & 659 & 195 & 66.5 & 02 \\
\hline $23173+2600$ & 231722.7 & +260018 & 183 & 69.2 & 9.8 & 3.1 & 15 \\
\hline $23180+0838$ & $23 \quad 1801.2$ & +083845 & 31.7 & 10.4 & 1.7 & $<1.0(1)$ & -- \\
\hline $23201-1105$ & 232009.1 & -110530 & 41.6 & 17.4 & 3.0 & 1.2 & 15 \\
\hline $23213-4521$ & 232122.2 & -452129 & 125 & 73.1 & 13.1 & 6.0 & 28 \\
\hline $23257+1038$ & 232545.7 & +103808 & 186 & 112 & 23.1 & $6.7(1)$ & -- \\
\hline $23309+2213$ & 233057.1 & +221317 & 44.6 & 11.4 & 2.0 & $<1.4(1)$ & 18 \\
\hline $23412-1533$ & $2341 \quad 14.1$ & -153346 & 1240 & 395 & 50.2 & 14.8 & -- \\
\hline $23438+0312$ & 234350.4 & +031234 & 115 & 30.5 & 10.1 & 6.4 & -- \\
\hline $23522-0010$ & 235212.6 & $\begin{array}{llll}-00 & 10 & 10\end{array}$ & 31.8 & 8.2 & 1.4 & $<1.0$ & 18 \\
\hline $23551+2451$ & 235511.4 & +245143 & 33.3 & 8.5 & 1.4 & $<0.9$ & 18 \\
\hline 23564-5651 & 235629.4 & $-5651 \quad 16$ & 69.6 & 29.3 & 3.8 & 2.0 & 02 \\
\hline $23575+2536$ & 235733.2 & +253629 & 45.0 & 16.6 & 2.7 & 1.6 & 15 \\
\hline 23594-0617 & 235924.4 & -061731 & 59.9 & 13.3 & 2.2 & $<1.0$ & 18 \\
\hline
\end{tabular}

Notes:

(1) Significant contamination of $100 \mu \mathrm{m}$ flux by local cirrus is possible, i.e., $\mathrm{c} 1>3$ or $\mathrm{c} 2>4$, see the Supplement.

(2) Probability of variability at $12 \mu \mathrm{m}$ and $25 \mu \mathrm{m}>90 \%$.

(3) Source is extended at $60 \mu \mathrm{m}$ and $100 \mu \mathrm{m}$. See text for method of flux density determination at these wavelengths.

(4) Source was surveyed with only a single hours-confirmation. 
flagged in Table I. The uncertainties on these corrected fluxes are on the order of $20 \%$. No sources showed extended emission at $12 \mu \mathrm{m}$ or $25 \mu \mathrm{m}$.

The last column of Table I gives the classification of the low resolution, $8 \mu \mathrm{m}$ to $22 \mu \mathrm{m}$ spectra of these sources, if available. These data were obtained with the IRAS spectrometer and were classified by assigning a two-digit number to all spectra. The first digit describes the gross shape of the spectrum, while the second digit provides a quantitative index of a specific feature in the spectrum. Values of the leading digit have the following meaning: " 1 " denotes a specutrum of a star with spectral type earlier than M5; "2" denotes the spectrum of an oxygen-rich star with an optically thin shell; " 3 " denotes the spectrum of a star with an optically thick oxygen-rich shell; "4" denotes the spectrum of a carbon-rich star with a shell; "0" denotes an unclassifiable or low signal-to-noise spectrum; " 8 " denotes the presence of the $11.3 \mu \mathrm{m}$ emission line.

\section{Identifications}

Table II includes identifications of the bright $12 \mu \mathrm{m}$ IRAS sources with objects in a number of astronomical catalogs. The term "identification" is used here rather than the term "association" used in the IRAS Catalog, since the extra work required to confirm a loose positional association has, in this case, been performed. Most of the identifications were made with objects in the SAO, IRC, or RAFGL catalogs. Most identifications were located within $90^{\prime \prime}$ of the IRAS position, although a few RAFGL identifications were located as far away as $3^{\prime}$ and are marked by footnotes. In Table II the first column gives the IRAS source name from Table I. The second column gives the corresponding source in the RAFGL. The third column gives the number of the corresponding source in the IRC. The fourth column gives the spectral type from the RAFGL, where available, and in other cases from the SAO, the General Catalog of Variable Stars (GCVS, Kukarkin et al. 1965), the New Catalog of Suspected Variable Stars (NSV, Kukarkin et al. 1982) the Carbon Star Catalog (Stephenson 1973), or the Catalog of Stellar Identifications (CSI, Ochsebein, Bischoff, and Egret 1979). The fifth column contains the $(2.2 \mu \mathrm{m}-12 \mu \mathrm{m})$ color indices where the $2.2 \mu \mathrm{m}$ magnitudes have been taken from the IRC. There exist $2.2 \mu \mathrm{m}$ magnitudes for several more of the stars in Table II (see, e.g., compilation by Gezari, Schmitz, and Mead 1984) but they are not used here as they do not form a homogeneous set with the IRC magnitudes. The infrared magnitudes used here are defined using a 10,000 K blackbody subtending a solid angle of $1.57 \times 10^{-16}$ steradians as a zero magnitude reference. Such an object would have flux densities of $28.3,6.73$, 1.19 , and $0.43 \mathrm{Jy}$ at $12,25,60$, and $100 \mu \mathrm{m}$, respectively. The last column contains the source name as given in the GCVS, RAFGL, SAO, CSI, NSV, and other comments.

\section{Discussion}

The vast majority of bright $12 \mu \mathrm{m}$ sources have been identified with stars. Based on these identifications, 208 $(77 \%)$ of the 271 sources are M stars, $24(9 \%)$ are carbonrich stars including $\mathrm{R}$ Coronae Borealis, $16(6 \%)$ are $\mathrm{K}$ stars, and $3(1 \%)$ are S stars. Seventeen $(7 \%)$ have no observed optical spectral type, but lie in the areas of the color-color diagrams, discussed below, inhabited by stars. Eleven of these 17 were associated with cataloged stars. The remaining two objects in the sample are the galaxies M82 and NGC 1068.

Infrared color indices of the sample are shown in Figures 1 and 2. The infrared indices of M 82 and NCC 1068 are significantly different from the indices of the other 269 sources in the sample, being typical of infrared active spiral galaxies (Soifer et al. 1984) and are not included in the figures.

The color indices in Figures 1 and 2 clearly differentiate between the stars with circumstellar dust shells and those without shells. An obvious feature of the figures is the marked clustering of $\mathrm{K}$, early- $\mathrm{M}$, and some late-M stars in the region expected for photospheric temperatures $2000 \mathrm{~K}-5000 \mathrm{~K}$. A second group of M stars shows a strong infrared excess at $12 \mu \mathrm{m}$ and $25 \mu \mathrm{m}$ due to cool $(<$ $200 \mathrm{~K}-300 \mathrm{~K}$ ) circumstellar dust shells. The onset of a substantial $12 \mu \mathrm{m}$ and $25 \mu \mathrm{m}$ excess occurs rather abruptly at spectral types later than M3-M4. There does not appear to be a continuous distribution in the amount of $[12 \mu \mathrm{m}]-[25 \mu \mathrm{m}]$ excess; rather there is a gap between stars on the photospheric line with a negligible excess and those with excesses greater than $0.3 \mathrm{mag}$ in $[12 \mu \mathrm{m}]-$ [25 $\mu \mathrm{m}]$. If there is an evolutionary sequence from stars with no shells to stars with shells, then this gap suggests that the observed dust shells form on time scales that are short compared to the time that the shell remains detectable in the infrared. Finally, a population consisting mostly of carbon stars shows an infrared excess from colder $(50 \mathrm{~K}-150 \mathrm{~K})$ material than surrounds many of the M stars (Fig. 1).

The spectral energy distributions of the stars with shells are generally consistent with models for such shells (Rowan-Robinson and Harris 1983). The late-type M stars show excesses associated with the silicate absorption bands at $10 \mu \mathrm{m}$ and $20 \mu \mathrm{m}$. A small number of stars, typically classified as carbon-rich stars, do follow the blackbody line at $2.2,12$, and $25 \mu \mathrm{m}$ down to temperatures as low as $500 \mathrm{~K}$.

As mentioned above, spectral types have not been previously determined for 17 of the sources in the sample. The $12 \mu \mathrm{m}-25 \mu \mathrm{m}-60 \mu \mathrm{m}$ colors of 13 of these sources are consistent with $\mathrm{M}$ stars with circumstellar dust shells. The colors of the five remaining sources place them in the region of overlap between $\mathrm{M}$ stars with circumstellar dust 
TABLE II

Catalog Comparison Data

\begin{tabular}{|c|c|c|c|c|c|}
\hline \multirow[b]{2}{*}{ Name } & Ider & tion & \multirow{2}{*}{$\begin{array}{l}\text { Spectral } \\
\text { Type } \\
\text { Assoc. }\end{array}$} & \multirow{2}{*}{$\begin{array}{c}{[2.2]-[12]} \\
(\mathrm{mag})\end{array}$} & \multirow[b]{2}{*}{ Notes } \\
\hline & RAFGL & IRC & & & \\
\hline$\overline{00050-2546}$ & $\overline{17}$ & -30002 & $\overline{\mathrm{M} 6 \mathrm{E}}$ & $\overline{3.1}$ & SY Scl \\
\hline 00121-1912 & 38 & -20006 & M1 III & 0.2 & $7 \mathrm{Cet}$ \\
\hline 00128-3219 & 40 & -30006 & M6E & 1.6 & $\mathrm{~S} \mathrm{Scl}$ \\
\hline 00192-2020 & 53 & -20007 & M5-6SE & 0.9 & T Cet \\
\hline 00193-4033 & 5017 & & & & (1) \\
\hline $00238-4234$ & 64 & & K0 & & $\alpha$ Phe \\
\hline $00245-652$ & 66 & -10009 & M7 & 1.6 & UY Cet \\
\hline $00254+1736$ & 71 & +20007 & M4 IIIB & 0.3 & 47 Psc \\
\hline 00254-3317 & 70 & & M3 & & $\eta \mathrm{Scl}$ \\
\hline 00254-1156 & $4032 \mathrm{~s}$ & -10010 & M4 & 0.2 & AG Cet \\
\hline 00410-1815 & 106 & -20010 & K1 III & 0.2 & $\beta$ Cet \\
\hline $00439+1512$ & 108 & +20012 & M4 IIIA & 0.2 & 57 Psc \\
\hline $00445+3224$ & 109 & +30015 & S6,2E & 2.1 & RW And \\
\hline 00515-6308 & & & MB & & HD 5276 \\
\hline $01030-3157$ & 156 & -30013 & M9 & 1.8 & AD Scl \\
\hline $01037+1219$ & 157 & +10011 & M10 & 5.7 & WX Psc \\
\hline $01085+3022$ & 168 & +30021 & M9 & 4.8 & NSV 426 \\
\hline $01217+2341$ & 5048 & +20023 & M7 & 1.5 & DO $8748(8)$ \\
\hline $01246-3248$ & 215 & -30015 & $\mathrm{C} 6,4$ & 1.8 & R Scl \\
\hline $01251+1626$ & 216 & +20025 & M6 & 1.8 & ST Psc \\
\hline 01261-4334 & 218 & & K5 & & $\gamma$ Phe \\
\hline $01438+1850$ & 5052 & +20029 & M6 & 1.8 & SV Psc \\
\hline $01452-8026$ & & & MB & & VZ Hyi \\
\hline $01516-4632$ & 261 & & M4 III & & $\psi$ Phe \\
\hline $01527+1656$ & 4013 & +20032 & M6 & 2.0 & DO $8984(8)$ \\
\hline 01576-2119 & 286 & -20024 & M1 G & 0.3 & o Cet \\
\hline 01579-0845 & 287 & -10030 & M3 III & 0.2 & AR Cet \\
\hline $02000+0726$ & 292 & +10025 & M7 & 1.4 & DO 358 (8) \\
\hline 02036-1027 & 297 & -10032 & M2 & 1.3 & UZ Cet \\
\hline $02043+2313$ & 5063 & +20038 & K2 IIIAB & 0.1 & $\alpha$ Ari \\
\hline $02168-0312$ & 318 & 00030 & M5.5E & & o Cet \\
\hline 02234-0024 & $4195 \mathrm{~s}$ & 00032 & $\mathrm{M} 4.5 \mathrm{E}$ & 3.0 & R Cet \\
\hline $02270-6944$ & & & M6-7 & & HD 15701 \\
\hline $02270-2619$ & 337 & -30021 & $\mathrm{C} 4,3 \mathrm{E}$ & 3.4 & R For \\
\hline $02351-2711$ & 357 & -30023 & M9 & 3.9 & NSV 878 \\
\hline 02401-0013 & $4220 \mathrm{~s}$ & & GALAXY & & NGC1068 (3) \\
\hline $02427-5430$ & & & $\mathrm{MC}$ & & W Hor \\
\hline $02455-1240$ & 378 & -10040 & M4 III & 1.0 & Z Eri \\
\hline $02455+1718$ & 379 & +20049 & M6E & 1.4 & T Ari \\
\hline $02464-5915$ & & & $\mathbf{M}$ & & X Hor \\
\hline $02497-0828$ & 392 & -10041 & M5 III & 0.9 & RR Eri \\
\hline $02522-5005$ & & & M7E G & & R Hor \\
\hline $02529+1807$ & & +20051 & M6 III & 0.3 & 45 Ari \\
\hline $02547+1106$ & 5087 & & & & \\
\hline
\end{tabular}


TABLE II (Continued)

\begin{tabular}{|c|c|c|c|c|c|}
\hline \multirow[b]{2}{*}{ Name } & & ion & \multirow{2}{*}{$\begin{array}{c}\text { Spectral } \\
\text { Type } \\
\text { Assoc. }\end{array}$} & \multirow{2}{*}{$\begin{array}{c}{[2.2]-[12]} \\
(\mathrm{mag})\end{array}$} & \multirow[b]{2}{*}{ Notes } \\
\hline & RAFGl & IRC & & & \\
\hline$\overline{02587+2136}$ & $\overline{414}$ & +20052 & M8 & 1.4 & UZ Ari \\
\hline $02596+0353$ & 419 & 00038 & M1.5 III & & $\alpha$ Cet \\
\hline $03082+1436$ & 455 & +10040 & M5.5E & 2.3 & U Ari \\
\hline $03112-5730$ & & & $\mathrm{C} 6,2.5$ & & TW Hor (4) \\
\hline $03172-2156$ & 475 & -20041 & M3 G & 0.2 & $\tau^{4}$ Eri \\
\hline $03287-1535$ & & & & & (2) \\
\hline 03318-1619 & 500 & -20043 & M7E & 1.8 & RT Eri \\
\hline 03336-7636 & & & $\mathrm{ME}$ & & $\mathrm{X}$ Men \\
\hline 03364-5533 & & & M6-7 III & & HD 22868 \\
\hline 03463-0710 & 525 & -10052 & M5 & 0.9 & BR Eri \\
\hline $03479-7423$ & & & M0 & & $\gamma \mathrm{Hyi}$ \\
\hline $03482-5213$ & & & M5-6 III & & HD 24306 \\
\hline 03489-0131 & & 00050 & M4 III & $1: 6$ & SU Eri \\
\hline 03505-0919 & & & & & $(2,5)$ \\
\hline $03507+1115$ & & +10050 & M8E & 4.2 & NML Tau \\
\hline $03511-45.58$ & & & ME & & U Hor \\
\hline $03557-1339$ & 537 & -10055 & M1 III & 0.1 & $\gamma$ Eri \\
\hline $04001-6217$ & & & MB & & $\gamma$ Ret \\
\hline $04020-1551$ & 542 & -20049 & M6 II & 1.9 & V Eri \\
\hline 04094-2515 & 552 & -30033 & M7E & 3.0 & W Eri \\
\hline $04140-8158$ & 4046 & & ME & & U Men \\
\hline 04157-1837 & 563 & -20052 & M7 & 1.7 & RS Eri \\
\hline 04311-0004 & & 00062 & M9 & 2.1 & BD Eri \\
\hline 04330-6307 & & & M4E & & R Ret \\
\hline $04345-2740$ & 605 & -30038 & M7 & 1.4 & UU Eri \\
\hline $04361-6210$ & & & M7 & & R Dor \\
\hline $04382-1417$ & 615 & -10075 & M7 & 1.8 & BX Eri \\
\hline 04382-1946 & 614 & -20059 & M4 G & 0.2 & DM Eri \\
\hline 04387-3819 & 617 & & M6E & & $\mathrm{R} \mathrm{Cae}$ \\
\hline 04404-7427 & & & & & SY Men \\
\hline 04573-1452 & 667 & -10080 & $\mathrm{C} 7,4 \mathrm{E}$ & 2.6 & R Lep \\
\hline $05027-2158$ & 682 & -20066 & M7E & 2.1 & T Lep \\
\hline $05033-2226$ & 688 & -20067 & K5 III & 0.2 & $\varepsilon$ Lep \\
\hline 05069-3434 & 699 & & $\mathrm{MC}$ & & HD 33452 \\
\hline $05071-6327$ & & & M3 III & & HD 33684 \\
\hline $05096-4834$ & & & ME & & S Pic \\
\hline $05098-6422$ & & & M7 & & U Dor \\
\hline $05217-3943$ & & & M0 & & SW Col \\
\hline $05418-4628$ & & & $\mathrm{~N}$ & & W Pic \\
\hline $08063+6522$ & 1232 & +70082 & M5-6 G & 1.3 & RZ UMa \\
\hline $08525+1725$ & 1298 & +20206 & $\mathrm{C} 5,4$ & 1.2 & X Cnc \\
\hline $08538+2002$ & 1301 & +20207 & $\mathrm{C} 5,5$ & 1.8 & T Cnc \\
\hline $08555+1102$ & 1302 & +10199 & M4 III & 1.0 & RT Cnc \\
\hline $09057+1325$ & 1321 & +10203 & M6 & 1.3 & CW Cnc \\
\hline $09069+2527$ & 1323 & +30208 & M7E III & 2.3 & W Cnc \\
\hline
\end{tabular}


TABLE II (Continued)

\begin{tabular}{|c|c|c|c|c|c|}
\hline \multirow[b]{2}{*}{ Name } & & ion & \multirow[t]{2}{*}{$\begin{array}{l}\text { Spectral } \\
\text { Type }\end{array}$} & \multirow{2}{*}{$\begin{array}{c}{[2.2]-[12]} \\
(\mathrm{mag})\end{array}$} & \multirow[b]{2}{*}{ Notes } \\
\hline & RAFGl & IRC & & & \\
\hline$\overline{09076+3110}$ & 1326 & +30209 & M6 IIIAS & 1.2 & "RS Cnc \\
\hline $09180+3436$ & 1341 & +30210 & K IIIAB & 0.1 & $\alpha$ Lyn \\
\hline $09180+5654$ & 1344 & +60193 & M4 IIIA & 0.4 & CG UMa \\
\hline $09425+3444$ & 1376 & +30215 & M7E & 2.1 & R Lmi \\
\hline $09430+5721$ & 1378 & +60197 & M3 IIIAB & 0.4 & CS UMa \\
\hline $09448+1139$ & 1380 & +10215 & M7E & & R Leo \\
\hline $09452+1330$ & 1381 & +10216 & C6 & 9.2 & CW Leo \\
\hline $09517+6954$ & 1388 & & GALAXY & & M 82 (3) \\
\hline $10131+3049$ & 1403 & +30219 & $\mathrm{CE}$ & 6.1 & RW LMi \\
\hline $10172+2005$ & 1410 & +20219 & K0 III & 0.1 & $\gamma^{1}$ Leo \\
\hline $10193+4145$ & 1411 & +40218 & M0 III & 0.2 & $\mu \mathrm{m}$ UMa \\
\hline $10305+7001$ & 1423 & +70095 & M6 & 1.5 & CT UMa \\
\hline $10350-1307$ & 1427 & -10242 & $\mathrm{C} 6,4$ & 1.2 & U Hya \\
\hline $10353-1145$ & 1428 & -10243 & M6 & 1.5 & FF Hya \\
\hline $10411+6902$ & 1432 & +70099 & M5E & 2.9 & R UMa \\
\hline $10416+6740$ & 1433 & +70100 & $\mathrm{C} 6,3$ & 0.9 & VY UMa \\
\hline 10491-2059 & 1439 & -20218 & $\mathrm{C} 6,5$ & 3.4 & V Hya \\
\hline $10521+7208$ & 1443 & +70102 & M8E III & 2.8 & VX UMa \\
\hline $10580-1803$ & 1450 & -20222 & M7 & 2.0 & R Crt \\
\hline $11006+6201$ & 1454 & +60208 & K0 IIIA & 0.1 & $\alpha \mathrm{UMa}$ \\
\hline $11125+7524$ & 1474 & +80023 & M5 & 1.8 & CS Dra \\
\hline $11251+4527$ & 1489 & +50211 & M4 III & 0.9 & ST UMa \\
\hline $11252+1525$ & 1488 & +20229 & M5 & 1.9 & AF Leo \\
\hline $11284+6936$ & 1494 & +70107 & M0 III & 0.1 & $\lambda$ Dra \\
\hline $11308-1020$ & & & & & $(2)$ \\
\hline $11358+0824$ & 1502 & +10243 & M4 III & 0.2 & $\omega \mathrm{Vir}$ \\
\hline $11432+0648$ & 1509 & +10245 & M1 IIIAB & 0.1 & $v$ Vir \\
\hline $11445+4344$ & 1511 & +40228 & M6 III & 1.8 & AZ UMa \\
\hline $11462-2628$ & 1512 & -30182 & M4 G & 0.2 & II Hya \\
\hline $11473-2718$ & 1515 & -30183 & M5 & 1.3 & HD 102766 \\
\hline $11485-1055$ & 1516 & -10258 & M3 & 1.1 & RU Crt \\
\hline 11501-0719 & 4830s & -10259 & M6-7E & 1.2 & S Crt \\
\hline $11538+5808$ & 1519 & +60213 & M6E & 1.6 & Z UMa \\
\hline $12020+0254$ & & 00214 & MA & 2.6 & TZ Vir \\
\hline $12046-0629$ & 1535 & -10263 & M5 III & 1.2 & RW Vir \\
\hline $12075-2220$ & 1536 & -20233 & K2 III & 0.2 & $\varepsilon \mathrm{CRv}$ \\
\hline $12226+0102$ & 1549 & 00217 & $C 5,3 E$ & 1.7 & SS Vir \\
\hline $12277+0441$ & 1554 & 00220 & M7 III & 1.3 & BK Vir \\
\hline $12344+2720$ & 1564 & +30241 & M5 & 1.8 & DO $14615(8)$ \\
\hline $12345-1715$ & 1565 & -20242 & M6 & 2.3 & T Crv \\
\hline $12380+5607$ & 1570 & +60220 & M7 II & 1.4 & Y UMa \\
\hline $12427+4542$ & 1576 & +50219 & $\mathrm{C} 5,5$ & 1.3 & Y CVn \\
\hline $12447+0425$ & 1579 & 00224 & $\mathrm{C} 8,1 \mathrm{E}$ & 4.3 & RU Vir \\
\hline $12517-0915$ & 1583 & -10274 & M3 III & 0.3 & $\psi$ Vir \\
\hline $12526+4728$ & 1585 & +50222 & M5 III & 0.3 & TU CVn \\
\hline $12530+0340$ & 1586 & 00226 & M3 III & 0.3 & $\delta$ Vir \\
\hline
\end{tabular}


TABLE II (Continued)

\begin{tabular}{|c|c|c|c|c|c|}
\hline \multirow[b]{2}{*}{ Name } & \multicolumn{2}{|c|}{ Identification } & \multirow{2}{*}{$\begin{array}{c}\text { Spectral } \\
\text { Type } \\
\text { Assoc. }\end{array}$} & \multirow{2}{*}{$\begin{array}{c}{[2.2]-[12]} \\
(\mathrm{mag})\end{array}$} & \multirow[b]{2}{*}{ Notes } \\
\hline & RAFGl & IRC & & & \\
\hline$\overline{12544+6615}$ & 1588 & +70116 & $\overline{\mathrm{C} 4,5}$ & 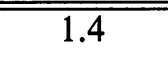 & RY Dra \\
\hline $12562+2324$ & 5278 & & M2 & & $\mathrm{T}$ Com (1) \\
\hline $13001+0527$ & 1594 & +10262 & M8 III & 1.9 & RT Vir \\
\hline $13039+2253$ & 5282 & +20254 & M5 IIIA & 0.3 & 40 Com \\
\hline $13114-0232$ & 1606 & 00230 & M7 III & & SW Vir \\
\hline $13172+4547$ & 1615 & +50226 & M6 IIIA & 2.6 & V CVn \\
\hline $13269-2301$ & 1627 & -20254 & M6.5E & & R Hya \\
\hline $13303-0656$ & 1633 & -10290 & M6.5E & 2.1 & S Vir \\
\hline $13462-2807$ & 1650 & -30207 & M8E & & W Hya \\
\hline $13468+3947$ & 1652 & +40248 & M6.5E & 1.9 & R CVn \\
\hline $13492-0325$ & 1653 & 00237 & M6 & 1.2 & AY Vir \\
\hline $13495+3441$ & 1654 & +30251 & M3 III & 0.3 & AW CVn \\
\hline $13499+6458$ & 1656 & +60226 & M3 IIIAS & 0.2 & 10 Dra \\
\hline $13582+3806$ & $4924 s$ & +40252 & M8 & 1.5 & DO $14839(8)$ \\
\hline $14059+4405$ & 1680 & +40253 & M4 III & 0.3 & BY Boo \\
\hline $14086-0730$ & 1686 & & M9 III & & \\
\hline $14086-2839$ & 1685 & -30215 & M6.5E & 2.5 & RU Hya \\
\hline $14133+1925$ & 1693 & +20270 & K2 IIIP & & $\alpha$ Boo \\
\hline $14142-1612$ & 1694 & -20266 & M6 III & 1.4 & EW Vir \\
\hline $14162+6701$ & 1696 & +70124 & M6E & 1.5 & U UMi \\
\hline $14219+2555$ & 1706 & +30257 & M8E III & & RX Boo \\
\hline $14247+0454$ & 1710 & 00243 & M6E III & 2.8 & RS Vir \\
\hline $14277+3904$ & $4947 \mathrm{~s}$ & +40257 & M6E & 1.1 & V Boo \\
\hline $14371+3245$ & 1719 & +30261 & M5E & 1.3 & RV Boo \\
\hline $14390+3147$ & 1720 & +30262 & M5 G & 1.0 & RW Boo \\
\hline $14412+2644$ & 1724 & +30263 & M3 IIIA & 0.2 & 34 Boo \\
\hline $14427+2717$ & 4201 & +30264 & K1 III & 0.1 & $\varepsilon$ Boo \\
\hline $14437+1520$ & 1728 & +20275 & M5 IIAB & 0.4 & DO 15069 (8) \\
\hline $14508+7421$ & 1740 & +70125 & K4 III & 0.2 & $\beta \mathrm{UMi}$ \\
\hline $14550-1214$ & & -10308 & M5 G & 1.3 & FY Lib \\
\hline $14567+6607$ & 1744 & +70126 & M5 III & & RR UMi \\
\hline $15060+0947$ & & & & & $(2)$ \\
\hline $15193+1429$ & 1765 & +10290 & M5E III & 1.8 & S Ser \\
\hline $15193+3132$ & 4990s & +30272 & M6.5E & 1.8 & $\mathrm{~S} \mathrm{CrB}$ \\
\hline $15223-0203$ & 1769 & 00265 & M7 & 1.4 & DO $3724(8)$ \\
\hline $15255+1944$ & 1773 & +20281 & M8.5E III & 4.2 & WX Ser \\
\hline $15262+0400$ & & 00266 & M9 & 3.8 & NSV 7098 \\
\hline $15298+0348$ & 1777 & 00268 & M8E III & 2.0 & WW Ser \\
\hline $15314+7847$ & 1780 & +80030 & M7E III & 1.8 & S UMi \\
\hline $15341+1515$ & 1788 & +20282 & M5 IIB & 0.8 & $\tau^{4} \operatorname{Ser}$ \\
\hline $15361+2441$ & 1790 & +20283 & M5 G & 0.5 & DO $15290(8)$ \\
\hline $15410-0133$ & 1793 & 00269 & M6E III & 1.7 & BG Ser \\
\hline $15418+0634$ & 1794 & +10294 & K2 IIIP & 0.1 & $\alpha \operatorname{Ser}$ \\
\hline $15464+1817$ & 1799 & +20284 & M1 IIIAB & 0.2 & K Ser \\
\hline $15465+2818$ & 4219 & & F8 IAP & & $\mathrm{R} \mathrm{CrB} \mathrm{(6)}$ \\
\hline
\end{tabular}


TABLE II (Continued)

\begin{tabular}{|c|c|c|c|c|c|}
\hline \multirow[b]{2}{*}{ Name } & \multicolumn{2}{|c|}{ Identification } & \multirow{2}{*}{$\begin{array}{l}\text { Spectral } \\
\text { Type } \\
\text { Assoc. }\end{array}$} & \multirow{2}{*}{$\begin{array}{c}{[2.2]-[12]} \\
(\mathrm{mag})\end{array}$} & \multirow[b]{2}{*}{ Notes } \\
\hline & RAFGl & IRC & & & \\
\hline$\overline{15477+3943}$ & & +40273 & $\overline{\mathrm{C} 6,3}$ & $\overline{3.1}$ & V CrB \\
\hline $15483+1517$ & 1801 & +20285 & M6.5E & 1.8 & R Ser \\
\hline $15492+4837$ & 5313 & +50246 & M6 IIIAS & 1.2 & ST Her \\
\hline $15566+3609$ & 5315 & +40276 & M7 & 2.3 & RS CrB \\
\hline $16011+4722$ & 5317 & +50248 & M6 G & 1.5 & X Her \\
\hline $16081+2511$ & 1832 & +30283 & M7E III & 2.2 & RU Her \\
\hline $16095+2337$ & 1834 & +20294 & M4 III & 0.3 & $10 \mathrm{Her}$ \\
\hline $16117-0334$ & 1837 & 00280 & M0.5 III & & $\delta \mathrm{Oph}$ \\
\hline $16164+5952$ & 1841 & +60241 & M4 IIIA & 0.3 & AT Dra \\
\hline $16235+1900$ & 1858 & +20298 & M7E & 2.6 & U Her \\
\hline $16260+3454$ & 1862 & +30292 & M9 & 3.9 & V697 Her \\
\hline $16269+4159$ & 1864 & +40283 & M6 III & & $30 \mathrm{Her}$ \\
\hline $16306+7223$ & 1868 & +70135 & M7E III & 1.4 & R UMi \\
\hline $16418+5459$ & 1886 & +50255 & M6 G & 1.6 & S Dra \\
\hline $16432+1213$ & 1889 & +10310 & M6E & 1.8 & UV Her \\
\hline $16473+5753$ & 1898 & +60248 & M7 & 1.2 & AH Dra \\
\hline $17081+6422$ & & +60249 & MS & 1.2 & TV Dra \\
\hline $17086+4045$ & & +40292 & M7 & 0.7 & DO $15828(8)$ \\
\hline $17133+3651$ & 1950 & +40295 & K3 II & 0.2 & $\pi$ Her \\
\hline $17190+2658$ & & & & & V393 Her \\
\hline $17329+5359$ & 1987 & +50267 & M7E III & 2.6 & SY Dra \\
\hline $17361+5746$ & 1993 & +60251 & M8 III & 1.8 & TY Dra \\
\hline $19510-5919$ & & & M7E & & S Pav \\
\hline $19575-5930$ & & & MB & & $v \mathrm{Pav}$ \\
\hline $20042-4241$ & 5578 & & & & V2334 Sgr \\
\hline $20075-6005$ & & & $\mathrm{MC}$ & & X Pav \\
\hline $20111-4708$ & & & ME & & R Tel \\
\hline $20120-4433$ & $7111 \mathrm{~s}$ & & $\mathrm{~S} 4,4 \mathrm{P}$ & & RZ Sgr (1) \\
\hline $20135-7152$ & & & & & NSV 12961 \\
\hline 20144-3916 & & & MD & & TT Sgr \\
\hline $20165-5051$ & 5585 & & MC & & Y Tel \\
\hline $20248-2825$ & 5587 & -30430 & M6E & & T Mic \\
\hline $20259-4035$ & 5588 & & M6E & & U Mic (1) \\
\hline 20296-2151 & $7118 \mathrm{~s}$ & -20590 & M9 & 2.9 & RU Cap \\
\hline 20359-3806 & 5589 & & & & NSV 13190 \\
\hline 20484-7202 & & & & & (2) \\
\hline $20526-5431$ & & & ME & & S Ind \\
\hline $20541-6549$ & & & & & (2) \\
\hline 21044-1637 & 2708 & -20596 & M6 III & 1.7 & RS Cap \\
\hline $21069-3843$ & 5592 & & & & \\
\hline $21100-1435$ & 2722 & -10558 & M5 & 1.1 & RX Aqr \\
\hline $21168-4514$ & 5593 & & No & & $\mathrm{T}$ Ind \\
\hline $21197-6956$ & & & $\mathrm{C} 7,3$ & & Y Pav \\
\hline $21206-4054$ & 5594 & & M6E & & V Mic \\
\hline $21243-6943$ & & & MB & & SX Pav \\
\hline
\end{tabular}


TABLE II (Continued)

\begin{tabular}{|c|c|c|c|c|c|}
\hline \multirow[b]{2}{*}{ Name } & & ion & \multirow{2}{*}{$\begin{array}{c}\text { Spectral } \\
\text { Type } \\
\text { Assoc. }\end{array}$} & \multirow{2}{*}{$\begin{array}{c}{[2.2]-[12]} \\
(\mathrm{mag})\end{array}$} & \multirow[b]{2}{*}{ Notes } \\
\hline & RAFGl & IRC & & & \\
\hline$\overline{21321+0136}$ & 2782 & $\overline{00504}$ & M6 & 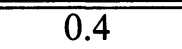 & $\overline{\text { DO } 7488(8)}$ \\
\hline $21368-3812$ & 5595 & & & & ISS 118 \\
\hline $21377-0200$ & 2787 & 00507 & M5 & 1.5 & DO $7540(8)$ \\
\hline $21417+0938$ & 2800 & +10503 & $\mathrm{~K} 2$ IB & 0.1 & $\varepsilon$ Peg \\
\hline $21439-0226$ & 2806 & 00509 & M7 III & 1.7 & EP Aqr \\
\hline $21453-4708$ & & & $\mathrm{ME}$ & & R Gru \\
\hline $21543-1421$ & 2819 & -10573 & M4 & 1.6 & SVS 5490 \\
\hline $22048+1138$ & 2851 & +10510 & M7 & 3.8 & SVS 102147 \\
\hline $22142-8454$ & & & M7 III & & HD 210548 \\
\hline $22145-8041$ & 4288 & & M6 III & & $\varepsilon$ Oct \\
\hline $22150-6030$ & & & K3 III & & $\alpha$ Tuc \\
\hline $22190-0751$ & 2889 & -10580 & MB & 1.4 & DZ Aqr \\
\hline $22196-4612$ & 4289 & & $\mathrm{~S} 4,7$ & & $\pi^{1} \mathrm{Gru}$ \\
\hline $22230-4841$ & $4687 \mathrm{~s}$ & & M8E III & & S Gru (1) \\
\hline $22231-4529$ & 5603 & & & & (1) \\
\hline $22267-4400$ & 5604 & & M4 & & $\delta^{2} \mathrm{Gru}$ \\
\hline $22280+1250$ & $5694 \mathrm{~s}$ & +10519 & M6EP & 1.3 & GM Peg \\
\hline $22296-6214$ & & & MB & & $v$ Tuc \\
\hline $22359-1417$ & $5702 s$ & -10584 & M7 & 1.2 & AB Aqr \\
\hline $22396-4708$ & 4292 & & M3 II & & $\beta$ Gru \\
\hline $22516+0838$ & 2984 & +10523 & M9 III & 2.9 & DO $7912(8)$ \\
\hline $22525-2952$ & 2989 & -30456 & MB & 1.5 & V Psa \\
\hline $22540-5740$ & 4293 & & M8 III & & HD 216824 \\
\hline $22596+1019$ & 4295 & +10525 & M9.5 & 4.3 & \\
\hline $23041+1016$ & 3023 & +10527 & M7E III & 2.1 & R Peg \\
\hline $23063-3024$ & 3029 & -30465 & M4 & 1.7 & Y Scl \\
\hline $23070+0824$ & 3031 & +10529 & M4S & 0.3 & GZ Peg \\
\hline $23086+0443$ & 3039 & +00527 & M5 G & 0.4 & DO 7959 (8) \\
\hline $23134-7031$ & & & $\mathrm{MC}$ & & HD 219831 \\
\hline $23142-0759$ & 3058 & -10597 & M3 III & 0.3 & $\chi$ Aqr \\
\hline $23142+1019$ & 3059 & +10531 & M7 & 0.4 & EO Peg \\
\hline $23166+1655$ & 3068 & & $\mathrm{C}$ & & \\
\hline $23173+2600$ & 3075 & +30509 & M7E III & 1.7 & W Peg \\
\hline $23180+0838$ & 3076 & +10533 & M6.5E III & 1.7 & S Peg \\
\hline $23201-1105$ & 3083 & -10598 & M8 & 1.5 & SV Aqr \\
\hline $23213-4521$ & 4296 & & & & SVS 5712 \\
\hline $23257+1038$ & 3099 & & $\mathrm{C}$ & & \\
\hline $23309+2213$ & 3113 & +20550 & M5 IIIA & 0.2 & $71 \mathrm{Peg}$ \\
\hline $23412-1533$ & 3136 & -20642 & M7E PEC & 2.9 & R Aqr (7) \\
\hline $23438+0312$ & 3147 & +00532 & $\mathrm{C} 7,2$ & 0.8 & 19 Psc \\
\hline $23522-0010$ & 3174 & -00535 & M5 IIB & 0.3 & XZ Psc \\
\hline $23551+2451$ & 3186 & +20557 & M3 III & 0.0 & $\psi \mathrm{Peg}$ \\
\hline $23564-5651$ & & & M3E & & S Phe \\
\hline $23575+2536$ & 3194 & +30522 & M7E III & 1.8 & Z Peg \\
\hline $23594-0617$ & 3197 & -10608 & M3 III & 0.5 & 30 Psc \\
\hline
\end{tabular}


TABLE II (Continued)

Notes:

1) Positions discrepant with RAFGL, but identifications likely.

2) Not previously cataloged.

3) Extended source. Point source flux densities quoted may be misleading.

4) Confused with a galaxy at $100 \mu \mathrm{m}$.

5) SW Eri is nearby.

6) Hydrogen deficient, carbon rich star.

7) Mira star with hot sub dwarf companion and radio jet (Kafatos, Hollis, and Michalitsianos, 1983).

8) Dearborn Observatory Catalog of red stars.
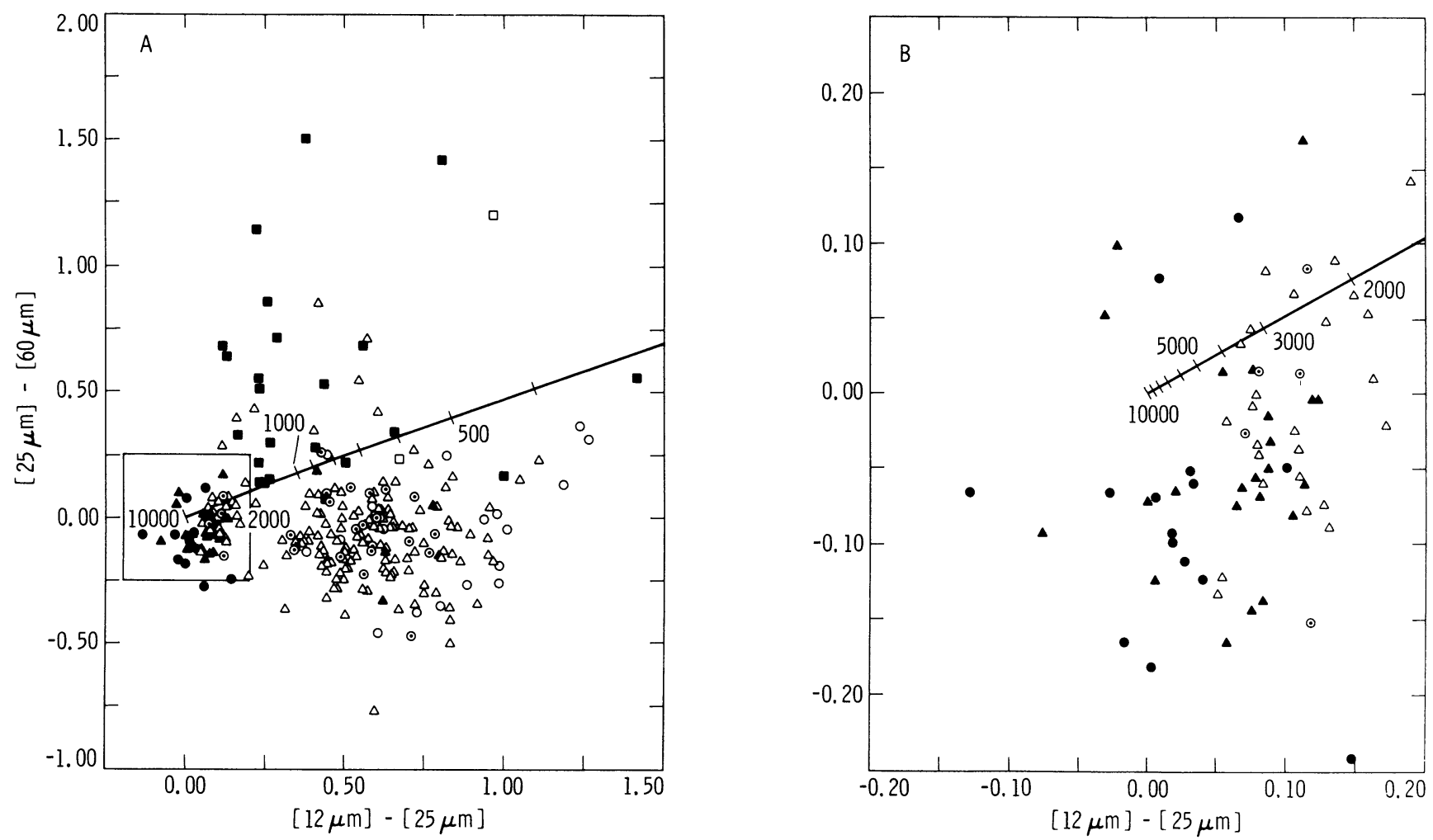

FIG. 1-The IRAS $12 \mu \mathrm{m}-25 \mu \mathrm{m}-60 \mu \mathrm{m}$ color indices are shown for all stars in the sample. The magnitude scale is defined in the text. Figure $1 \mathrm{~B}$ is an enlargement of the box shown in Figure 1. K stars are represented as filled circles. M0-M3 stars are represented as filled triangles, M4-M10 stars are represented as open triangles, $\mathrm{M}$ stars with no subtype are represented as dots within a circle, $\mathrm{S}$ stars are represented as open squares, carbon stars (including $\mathrm{R} \mathrm{CrB}$ ) are represented as filled squares, and stars with no previously cataloged spectral types are represented as open circles. See text for sources of spectral types.

shells and carbon stars in Figure 1.

The spectra of all of the 17 sources without previously cataloged spectral types were measured with the IRAS spectometer. Of these, 15 have silicate emission features and IRAS colors consistent with their being $\mathrm{M}$ stars with circumstellar dust shells, while two show the emission feature at $11 \mu \mathrm{m}$ characteristic of carbon stars.

Stars with no spectral features and stars with optically thin oxygen-rich dust shells (classes $1 n$ and $2 n$, respectively) each comprise about $40 \%$ of those whose spectra were measured. In general, the strength of the silicate emission features correlates with the $[12 \mu \mathrm{m}]-[25 \mu \mathrm{m}]$ excess. That is, the sources with the strongest silicate emission features tend to be the late-M stars toward the right side in Figures 1 and 2. The IRAS spectral classifications also agree well with the optical spectral types. Only 

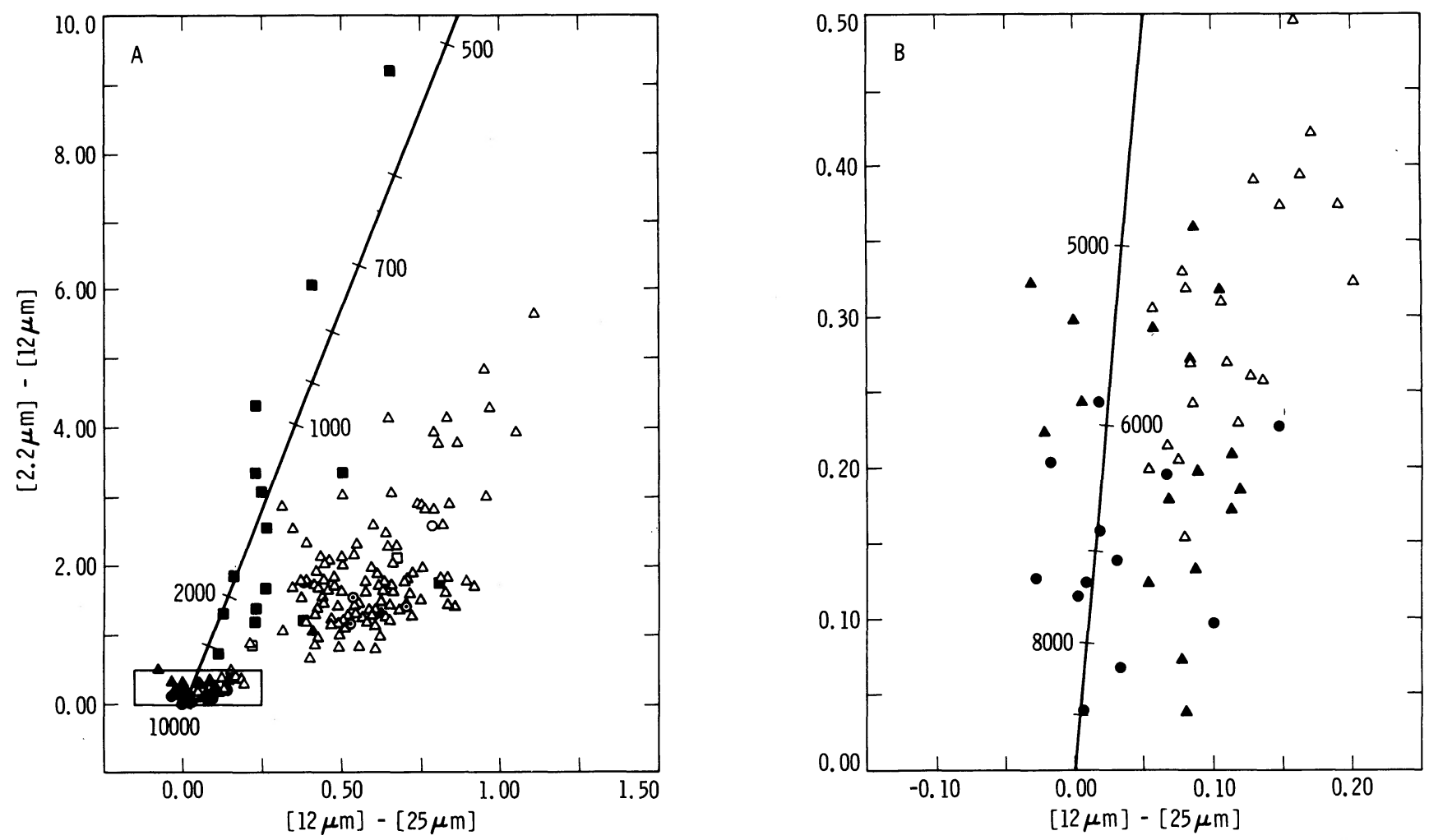

FIG. 2-The $2.2 \mu \mathrm{m}-12 \mu \mathrm{m}-25 \mu \mathrm{m}$ color indices are shown for all sources in the sample that have counterparts in the Two Micron Sky Survey Catalog (Neugebauer and Leighton 1969). The $2.2 \mu \mathrm{m}$ magnitudes were taken from the Two Micron Sky Survey Catalog and the $12 \mu \mathrm{m}$ and $25 \mu \mathrm{m}$ magnitudes are taken from the IRAS data (see Table II). Symbol notation is the same as in Figure 1.

five stars gave possibly conflicting classifications. A visual examination of the IRAS spectra of $08555+1102$ (RT Canari M4 III), $15492+4837$ (St Herculis, M6 III $a$ s), and 22196-4612 ( $\pi$ Gruis, S4,7) indicated that weak silicate features are present in the infrared spectra rather then weak silicon carbide emission as suggested by the IRAS classification. The infrared spectrum of 21197-6956 ( $\gamma$ Pavonis, $\mathrm{C7}, 3$ ) is featureless, showing no sign of the silicon carbide or silicate features.

The remaining discrepant case $1349+3441$ (AW Canum Venaticorum, M3 III) is quite interesting because the $11.3 \mu \mathrm{m}$ feature is clearly present in its infrared spectrum. One successful model for the $11.3 \mu \mathrm{m}$ feature (Leger and Puget 1984) suggests that it is caused by emission from small grains formed primarily of carbon. Such grains are not likely, however, to be formed in the oxygen-rich environment implied by the classification of this star. If this feature is like that seen in other environments such as planetary nebulae, and is associated with AW CVn, then either the spectral classification of this star is incorrect, or the model of small carbon grains as the carriers of the unidentified infrared features may have to be modified.

Given that the sample is composed of late-type stars, one expects some of the sources to be variable. Thirtyfour sources are listed in the IRAS Point Source Catalog with a probability greater than $90 \%$ of being variable at 12 $\mu \mathrm{m}$ and $25 \mu \mathrm{m}$. (Supplement). These sources are flagged in Table I. Most of the variables have $12 \mu \mathrm{m}-25$ $\mu \mathrm{m}-60 \mu \mathrm{m}$ colors consistent with a star with a circumstellar dust shell; those with a known spectral type are late-M or carbon stars. Typically, these sources varied by a factor of 1.5-2.0 over a period of about six months at $12 \mu \mathrm{m}$ with the amplitude diminishing with increasing wavelength.

\section{Summary}

Almost all sources brighter than 0 magnitude at $12 \mu \mathrm{m}$ with absolute galactic latitude greater than $30^{\circ}$ have infrared colors typical of late-type stars and may be identified as such in star catalogs or by their infrared colors and spectra. Twelve sources exhibited extended emission at $60 \mu \mathrm{m}$ and $100 \mu \mathrm{m}$, one source (AW CVn) has the $11.3 \mu \mathrm{m}$ feature present in its spectrum, and 34 sources are probably variable in the far infrared. $\mathrm{K}$ and $\mathrm{M}$ stars without circumstellar dust shells, $M$ stars with circumstellar dust shells, and carbon stars occupy well-defined regions of infrared color-color diagrams and have distinctive $8 \mu \mathrm{m}$ to $22 \mu \mathrm{m}$ spectra. These data will be useful in classifying unidentified IRAS sources.

We thank H. H. Aumann for useful discussions, Sharon Conrad and Rosanne Hernandez for their help and pa- 
tience with the text and tables, and Judy Bennett, Joe Chillemi, and Ted Sesplaukis for helping the data flow smoothly.

\section{REFERENCES}

Fowler, J. W., and Rolfe, E. G. 1982, Journal of the Astronautical Sciences 30, 385.

Gezari, D. Y., Schmitz, M., and Mead, J. M. 1984, Catalog of Infrared Observations NASA Reference Publication 1118.

IRAS Explanatory Supplement, 1984, C. A. Beichman, G. Neugebauer, H. Habing, P. Clegg, and T. Chester, eds. (Jet Propulsion Laboratory preprint D-1855).

Jung, and Bischoff,. 1979, General Catalog of Stellar Identifications Strasbourg: Centre des Donnees Stellaires )

Kafatos, M., Hollis, J. M., and Michalitsianos, A. G., 1983, Ap. J. (Letters) 267, L103.
Kukarkin, B. V. et al. 1965, General Catalog of Variable Stars (3rd edition), (Moscow: Nauka).

Kukarkin, B. V. et al. 1982, New Catalog of Suspected Variable Stars (Moscow: Nauka).

Leger, A., and Puget, J. L. 1984, Astr. and Ap. (submitted)

Low, F. et al. 1984, Ap. J. (Letters) 278, L19.

Neugebauer, G. and Leighton, 1969, Two Micron Sky Survey Catalog, NASA SP-3047.

Neugebauer, G. et al., 1984 Ap. J. (Letters) 278, L1.

Price, S. P., and Murdock, T. L. 1983, The Revised AFGL Infrared Sky Survey Catalog, AFGL-TR-83-0161.

Rowan-Robinson, M., and Harris, S. 1983, M.N.R.A.S. 202, 797.

Smithsonian Astrophysical Observatory 1966, Smithsonian Astrophysical Observatory Star Catalog.

Stephenson, 1973, A General Catalog of Cool Carbon Stars, Pub. Warner and Swasey Observtory 1, No. 4.

Soifer, B. T. et al. 1984, Ap. J. (Letters) 278, L71. 